\title{
Guest Editorial to the Special Section on MODELS 2007
}

\author{
Gregor Engels
}

Published online: 24 November 2009

(C) Springer-Verlag 2009

This section of "Software \& Systems Modeling" contains four selected papers of the tenth MODELS conference. MODELS 2007 was held in Nashville, TN, USA, from September 30 to October 5, 2007. This conference on Model Driven Engineering Languages and Systems has established itself as the key international venue for the presentation of scientific results in the domain of model driven engineering and related topics such as software modeling and model transformation.

A total of 45 papers were selected for publication in the conference proceedings from 158 papers submitted for review. The papers presented at the conference covered a wide range of issues from model transformation, meta modeling, model and consistency analysis, service and process modeling, language definition, as well as modeling methods.

The program of the conference week comprised three keynote presentations, technical paper presentations, two panels, and several workshops and tutorials on key topics in the field. The invited speakers were Mary Shaw (Carnegie Mellon University), Kevin Sullivan (University of Virginia), and Patrick Lardieri (Lockheed Martin Advanced Technology Lab).

After the conclusion of the conference, the program committee was asked to propose a selection of the best papers for publication in a special issue of SoSyM. In the end, 13 papers were proposed, and their authors were invited to submit extended versions of the conference papers to this special section. All submitted papers underwent a full review process, and were evaluated by at least three reviewers, generally including both some of the original PC members and some fresh reviewers. Finally, four papers were selected for

G. Engels ( $ه)$

University of Paderborn, Warburger Str. 100,

33098 Paderborn, Germany

e-mail: engels@upb.de inclusion in this special section of SoSyM. They address current topics in the modeling community like model transformation and quality issues of models.

"Bidirectional model transformations in QVT: semantic issues and open questions" by Perdita Stevens discusses OMG's Queries, Views and Transformations (QVT) standard as applied to the specification of bidirectional transformations between models. In particular, it is shown that any transformation language sufficient to the needs of modeldriven development would have to be able to express nonbijective transformations.

"Comparing Relational Model Transformation Technologies-Implementing QVT with Triple Graph Grammars" by Joel Greenyer and Ekkart Kindler compares the concepts of the declarative languages of QVT and TGGs (Triple Graph Grammars). It turns out that TGGs and declarative QVT have many concepts in common. For instance, it is shown that QVT-Core can be implemented by transforming QVT-Core mappings to TGG-rules, which can then be executed by a TGG transformation engine that performs the actual QVT transformation.

"Automatic Generation of Basic Behavior Schemas from UML Class Diagrams" by Cristina Gómez, Manoli Albert, Jordi Cabot, and Vicente Pelechano presents a strategy for the automatic generation of a behavioral schema consisting of a set of system operations that the user may execute to update a system state. Operations in the schema are derived from the static aspects of the domain as defined in an UML class diagram, while taking into account possible dependencies among them to ensure completeness and executability.

"On Challenges of Model Transformation from UML to Alloy" by Kyriakos Anastasakis, Behzad Bordbar, Geri Georg, and Indrakshi Ray focuses on utilizing model transformation techniques to deal with the analysis of UML models and to identify design faults within a specification. It is 
shown how UML models can be automatically transformed into Alloy. The paper reports on the challenges of this model transformation, which are caused by fundamental differences in the design philosophy of UML and Alloy. 